\title{
Tobramycin Clearance Is Best Described by Renal Function Estimates in Obese and Non-obese Individuals: Results of a Prospective Rich Sampling Pharmacokinetic Study
}

\author{
Cornelis Smit ${ }^{1,2}$ - Roeland E. Wasmann ${ }^{3}$ Marinus J. Wiezer ${ }^{4} \cdot$ Hendricus P. A. van Dongen ${ }^{5}$. \\ Johan W. Mouton ${ }^{6}$ • Roger J. M. Brüggemann ${ }^{3}$ - Catherijne A. J. Knibbe ${ }^{1,2}$ (1)
}

Received: 7 April 2019 / Accepted: 22 May 2019 / Published online: 30 May 2019

(C) The Author(s) 2019

\begin{abstract}
Purpose Tobramycin is an aminoglycoside antibiotic of which the $24 \mathrm{~h}$ exposure correlates with efficacy. Recently, we found that clearance of the aminoglycoside gentamicin correlates with total body weight (TBW). In this study, we investigate the full pharmacokinetic profile of tobramycin in obese and non-obese individuals with normal renal function. Methods Morbidly obese individuals $(n=20)$ undergoing bariatric surgery and non-obese healthy volunteers $(n=8)$, with TBW ranging 57-194 $\mathrm{kg}$, received an IV dose of tobramycin with plasma concentrations measured over $24 \mathrm{~h}$ ( $n=10$ per individual). Statistical analysis, modelling and simulations were performed using NONMEM.
\end{abstract}

Results In a two-compartment model, TBW was the best predictor for central volume of distribution $(p<0.001)$. For clearance, MDRD (de-indexed for body surface area) was identified as best

Electronic supplementary material The online version of this article (https://doi.org/I 0. I007/s I | 095-0 | 9-265 I-2) contains supplementary material, which is available to authorized users.

Catherijne A. J. Knibbe

c.knibbe@antoniusziekenhuis.nl

Department of Clinical Pharmacy, St. Antonius Hospital, Koekoekslaan I, 3435, CM Nieuwegein, The Netherlands

2 Department of Systems Biomedicine and Pharmacology, Leiden Academic Centre for Drug Research

Leiden University, Leiden, The Netherlands

3 Department of Pharmacy

Radboud Institute for Health Sciences, Radboudumc

Nijmegen, The Netherlands

4 Department of Surgery

St. Antonius Hospital, Nieuwegein, The Netherlands

5 Department of Anesthesiology

St. Antonius Hospital, Nieuwegein, The Netherlands

6 Department of Medical Microbiology and Infectious Diseases, Erasmus MC, Rotterdam, The Netherlands covariate $(p<0.001)$, and was superior over TBW $((p<0.05)$. Other renal function estimates $(24 \mathrm{~h}$ urine GFR and de-indexed CKD-EPI) led to similar results as MDRD (all $p<0.001)$ ).

Conclusions In obese and non-obese individuals with normal renal function, renal function estimates such as MDRD were identified as best predictors for tobramycin clearance, which may imply that other processes are involved in clearance of tobramycin versus gentamicin. To ensure similar exposure across body weights, we propose a MDRD-based dosing nomogram for obese patients.

KEY WORDS aminoglycosides · morbid obesity . pharmacokinetics · tobramycin

\section{ABBREVIATIONS}

(pvc)VPC (prediction-variability corrected)

Visual Predictive Check

ABW Adjusted Body Weight

AUC Area Under the Curve

$\mathrm{AUC}_{24} 24 \mathrm{~h}$ Area Under the Curve

BMI Body Mass Index

BSA Body Surface Area

CG Cockcroft Gault

CKD-EPI Chronic Kidney Disease

Epidemiology Collaboration

GFR Glomerular Filtration Rate

GOF Goodness-of-fit

IBW Ideal Body Weight

IIV Inter-individual variability

LBW Lean Body Weight

MDRD Modification of Diet in Renal Disease

MIC Minimal Inhibitory Concentration

OFV Objective Function Value

TBW Total Body Weight 


\section{INTRODUCTION}

The global prevalence of obesity and morbid obesity, which is commonly defined as a body mass index (BMI) over 30 and $40 \mathrm{~kg} / \mathrm{m}^{2}$, respectively, is rapidly rising. In 2015 , over 600 million adults were obese worldwide, accounting for $12 \%$ of the entire adult population (1). Due to physiological changes associated with obesity, such as an increase in fat and other tissue, differences in liver size, liver flow, liver enzyme activity and glomerular filtration rate (GFR), obesity-related changes in pharmacokinetic (PK) and/or pharmacodynamic (PD) parameters of drugs may be expected (2). However, the exact quantification of these changes in PK and PD is lacking for many drugs. This is of particular relevance for drugs for which a target concentration and/or exposure related to efficacy or safety has been identified, like in the case of aminoglycosides. These antibiotics, such as gentamicin and tobramycin, are used for the treatment of severe infections, with their efficacy being closely related to a (timely) attainment of an adequate plasma exposure (depicted by the 24-h area under the curve $\left(\mathrm{AUC}_{24}\right)$ over the minimal inhibitory concentration (MIC) of the microbiological target (3-5). Since in the general population $\mathrm{AUC}_{24}$ closely correlates with the maximum plasma concentration $\left(\mathrm{C}_{\max }\right)$ and measurement of an AUC puts substantial burden to the treated patient, the $\mathrm{C}_{\max }$ is often used as measure of efficacy with target values between 15 and $20 \mathrm{mg} / \mathrm{L}$. Despite this approach that is used in clinical practice, the $\mathrm{AUC}_{24}$ is still considered the cornerstone PD-index for aminoglycoside effectivity and toxicity (5-7), with $75 \mathrm{mg} * \mathrm{~h} / \mathrm{L}$ being proposed as a pharmacodynamic target with an optimal effect and acceptable risk for toxicity (5). However, this is based on the assumption that MICs are not higher than $1 \mathrm{mg} / \mathrm{L}$, whereas the wild-type population of most gramnegatives extend to $2 \mathrm{mg} / \mathrm{L}(5,8)$.

To date, in clinical practice tobramycin is dosed on a $\mathrm{mg} / \mathrm{kg}$ basis. Clinicians may however be reluctant to use $\mathrm{mg} / \mathrm{kg}$ dosing in (morbidly) obese patients, since high trough levels (i.e. $>1 \mathrm{mg} / \mathrm{L} 24 \mathrm{~h}$ after dosing) are associated with side effects such as nephro- or ototoxicity $(9,10)$. Therefore, over the past decades, several alternative body size descriptors to guide aminoglycoside dosing have been proposed, such as adjusted body weight (ABW) and lean body weight (LBW) (11-16). These dosing measures were mainly proposed to compensate for a body weight-related increase in volume of distribution $\left(V_{d}\right)$ which was found in these studies (11-16), with $\mathrm{V}_{\mathrm{d}}$ being the parameter that determines $\mathrm{C}_{\max }$. However, since not $\mathrm{V}_{\mathrm{d}}$, but drug clearance drives the AUG, it is essential to clarify what body size descriptor or parameter best predicts clearance with increasing body weight. For the aminoglycoside gentamicin, we recently found that in obese individuals, TBW was the most predictive descriptor for clearance, albeit in a nonlinear manner (17). In the current prospective rich sampling study, we investigate the pharmacokinetics of tobramycin in morbidly obese and non-obese individuals with normal renal function (eGFR $>60 \mathrm{ml} / \mathrm{min}$ ), in order to investigate how tobramycin clearance and other PK parameters change in obesity. In line with our previous study on gentamicin PK in the obese, beside weight measures, other measures like renal function estimates were investigated as covariates. The results are used to guide dosing of tobramycin in (morbidly) obese individuals.

\section{MATERIALS AND METHODS}

This prospective observational study was registered in the Dutch Trial Registry (NTR6058), approved by the local human research and ethics committee and was conducted in accordance with the principles of the Declaration of Helsinki.

\section{Participants}

Morbidly obese patients $\left(\right.$ BMI $>40 \mathrm{~kg} / \mathrm{m}^{2}$ or $>35 \mathrm{~kg} / \mathrm{m}^{2}$ with comorbidities) scheduled for bariatric surgery (laparoscopic gastric sleeve or gastric bypass) were considered for inclusion. In addition, a group of non-obese healthy volunteers (body mass index (BMI) 18-25 $\mathrm{kg} / \mathrm{m}^{2}$ ) was included.

Participants were excluded when they had a known allergy to aminoglycosides, used potentially nephrotoxic medication in the week before surgery (such as lisdiuretics, vancomycin, ACE-inhibitors, non-steroid anti-inflammatory drugs), had a known renal insufficiency (eGFR $<60 \mathrm{ml} / \mathrm{min}$, using the Modification of Diet in Renal Disease (MDRD) (non-obese) or LBW in the Cockcroft Gault formula (obese) (18)), were pregnant or breastfeeding. Before inclusion, all participants provided written informed consent.

\section{Study Procedures}

Twenty morbidly obese patients received $5 \mathrm{mg} / \mathrm{kg} \mathrm{LBW}$ (calculated according to Janmahasatian (19)) tobramycin on the day of surgery as a single dose infused over $0.5 \mathrm{~h}$, after which venous blood samples were collected at $\mathrm{t}=5 \mathrm{~min}$ after end of infusion, followed by collections at $\mathrm{t}=1,1.5,2,2.5,3.5,4.5,6$, 12 and $24 \mathrm{~h}$ after start of infusion. $3 \mathrm{~mL}$ blood samples were collected in lithium-heparin tubes, centrifuged at $1900 \mathrm{~g}$ for $5 \mathrm{~min}$, and plasma was stored at $-80^{\circ} \mathrm{C}$ until analysis. Eight non-obese healthy volunteers received a single dose of $5 \mathrm{mg} / \mathrm{kg}$ TBW tobramycin, infused over $0.5 \mathrm{~h}$, after which the same sampling scheme was employed.

In order to measure the glomerular filtration rate (GFR), urine was collected over 24-h on the study day and before and $24 \mathrm{~h}$ after administration of tobramycin, a blood sample was 
collected to measure serum creatinine. In addition, GFR was estimated (eGFR) as follows: (1) using the Cockcroft-Gault formula with LBW without correction for gender for obese and with TBW for non-obese individuals (CG-LBW) (18), (2) using the Modification of Diet in Renal Disease (MDRD) which was de-indexed for body surface area (BSA) by multiplying with individual BSA/1.73, and (3) Chronic Kidney Disease Epidemiology Collaboration (CKD-EPI) formula, also de-indexed for body surface area (BSA) by multiplying with individual BSA/1.73 (18). Equations for the different renal function estimates are shown in the supplemental material.

Total tobramycin plasma concentrations were measured using a commercially available, validated immunoassay kit (Cobas ${ }^{\circledR}$ TOBR2, Roche Diagnostics GmbH, Mannheim), with a lower limit of quantification (LLOQ) of $0.3 \mathrm{mg} / \mathrm{L}$.

\section{Pharmacokinetic Analysis}

For each individual, $\mathrm{AUC}_{24}$ was calculated using the trapezoidal rule. $\mathrm{C}_{\max }$ was defined as the measured concentration $1 \mathrm{~h}$ after start of the 0.5 -h infusion. Categorical data was analysed using Fischer Exact test, where continuous data is compared using the Wilcoxon Rank test.

Using all data, population pharmacokinetic modelling was performed with NONMEM 7.3 (ICON Development Solutions, Hanover, USA), Pearl-speaks-NONMEM (PsN) 4.6.0 and visualized using Pirana 2.9.7 (Pirana Software \& Consulting BV), R 3.4.4 and GraphPad Prism 6.0 (GraphPad Software, La Jolla, USA) (20-22). Concentrations below LLOQ were retained in the dataset and analysed using the M3 method, where a likelihood for being below LLOQ was estimated for these concentrations (23). Discrimination between nested models was done by comparing the objective function value $(\mathrm{OFV},-2 \log$ likelihood) as obtained from the NONMEM output. A difference in $\mathrm{OFV}$ of 3.84 , corresponding with a $p$ value $<0.05$ for one degree of freedom, was considered statistically significant. In addition, goodness-of-fit plots (GOF, observed versus population and individual predicted values, individual weighted residuals versus time or population predicted values), predictionvariability corrected visual predictive checks (pvcVPG), precision of parameter estimates, shrinkage, and individual plots were examined for diagnostic purposes. One-, two- and threecompartment models were evaluated as structural models. Inter-individual variability (IIV) on the individual parameter estimate of the $i$ th individual $\left(\theta_{i}\right)$ was modelled according to Eq. (1):

$\theta_{i}=\theta_{\text {mean }} \times e^{\eta_{i}}$

where $\theta_{\text {mean }}$ is the population mean parameter value, $\eta_{i}$ is a random variable for the $i$ th individual with a mean of zero and variance of $\omega^{2}$, assuming log-normal distribution in the population. For residual variability a combined, proportional and additional error model was investigated, according to eq. (2):

$\Upsilon_{i j}=C_{\text {pred }, i j}+\left(C_{\text {pred }, i j} \times \varepsilon_{1}\right)+\varepsilon_{2}$

where $\mathrm{Y}_{i j}$ is the observed concentration, $\mathrm{C}_{p r e d, i j}$ the predicted concentration for the $j$ th observation in the $i$ th individual and $\varepsilon_{1}$ and $\varepsilon_{2}$ the proportional and additive errors, respectively, with a mean of zero and variance of $\sigma^{2}$.

\section{Covariate Analysis}

The influence of covariates was explored by plotting individual posthoc parameter estimates or the IIV estimates against individual covariate values. Covariates were TBW, LBW (calculated using the Janmahasatian formula) (19), ABW (calculated as ideal body weight (IBW) + $0.4 *$ (TBW-IBW) (11)), BMI, GFR, de-indexed MDRD, de-indexed CKD-EPI, GG-LBW, sex and age. Equations are summarized in the supplemental material. Continuous covariates were implemented using the following equations:

$P_{i}=P_{p} \times\left(\frac{C O V}{C O \mathrm{~V}_{\text {standard }}}\right)^{X}$

$P_{i}=P_{p} \times\left(I+Z \times\left(C O V-C O V_{\text {standard }}\right)\right)$

where $P_{\mathrm{i}}$ and $P_{\mathrm{p}}$ represent individual and population parameter estimates, $\mathrm{COV}$ represents the covariate, $\mathrm{COV}_{\text {standard }}$ represents a population standardized (e.g. $70 \mathrm{~kg}$ for TBW) or median value for the covariate, $\mathrm{X}$ represents the exponent for a power function and $\mathrm{Z}$ represents the relative change of the parameter in a linear covariate relationship. Linear covariate relationships were tested with a slope parameter $\mathrm{Z}$ using eq. (4) or by fixing the exponent $\mathrm{X}$ in eq. (3) to 1. In addition, the recently described function characterising the influence of TBW on gentamicin clearance (17), was evaluated for its performance for tobramycin (i.e. equation (3) using TBW as covariate with an exponent of 0.729 ), which is an approach that was applied before on aminoglycosides in neonates and children $(24,25)$. Categorical covariates were entered into the model by calculating a separate pharmacokinetic parameter for each category of the covariate. After entering covariates separately into the model, their added value was statistically tested using the OFV. In addition, if applicable, it was evaluated whether the IIV for the parameter decreased upon inclusion of the covariate and whether the trend in the IIV versus covariate disappeared. In general, a forward inclusion $(p<0.05$, OFV decrease $>3.8)$ and backward deletion $(p<0.001$, OFV decrease $>10.8)$ strategy was employed for inclusion of covariate. Finally, earlier mentioned general diagnostics were taken into account. 


\section{Internal Model Validation}

pvcVPC's were generated using $\mathrm{PsN}$ ( $n=1000$ datasets split for obese and non-obese) with prediction and variability correction. Bootstrap re-sampling $(n=1000$, stratified on weight group, i.e. obese and non-obese) was performed to obtain confidence intervals for the parameters, as well as to assess the robustness of the model.

\section{Model-Based Simulations}

Using the final PK model, Monte Carlo simulations were performed with interindividual and residual variability in 9.993 individuals with body weights uniformly distributed between 60 and $190 \mathrm{~kg}$. Values for de-indexed MDRD were assigned to each individual using a normal distribution with separate mean and standard deviation (SD) for obese (mean: $137 \mathrm{ml} / \mathrm{min}, \mathrm{SD}: 34$ ) and non-obese (mean: $112 \mathrm{ml} / \mathrm{min}, \mathrm{SD}$ : 23) groups, based on the distributions found in the ongoing AMIGO trial (Dutch Trial Registry NTR6058, $n=60$ obese, $n=32$ non-obese individuals,). Four dosing scenarios were simulated: (1) tobramycin $5 \mathrm{mg} / \mathrm{kg}$ TBW, (2) de-indexed MDRD based dosing using the relationship between clearance and MDRD as was found a-posteriori in the final PKmodel, with $75 \mathrm{mg}^{*} \mathrm{~h} / \mathrm{L}$ as target for the $\mathrm{AUC}_{24}$ (5) and (3) $5 \mathrm{mg} / \mathrm{kg}$ ABW. For comparison, (4) simulations using a dosing strategy based on the best function identified for TBW (Table III) were also performed. All infusions were simulated as single intravenous administrations given in $0.5 \mathrm{~h}$.

\section{RESULTS}

\section{Demographics and Data}

A total 20 obese and 8 non-obese participants were included in this study. Obese patients had a median TBW of $137.8 \mathrm{~kg}$ (range 103-194) versus $66.3 \mathrm{~kg}$ (range 57-91) in the non-obese group. Patient characteristics are shown in Table I. For each individual, 10 samples were obtained resulting in 280 tobramycin plasma concentrations in total. Of these, 23 $(8.2 \%)$ were below LLOQ of $0.3 \mathrm{mg} / \mathrm{L}$.

The mean measured tobramycin plasma concentrations for each timepoint are shown in Fig. 1. The $\mathrm{AUC}_{24}$ was significantly lower in the obese group receiving tobramycin as a single $5 \mathrm{mg} / \mathrm{kg} \mathrm{LBW}$ dose compared to the non-obese control group receiving a $5 \mathrm{mg} / \mathrm{kg}$ TBW dose (mean $56.1 \pm$ $16.3 \mathrm{mg}^{*} \mathrm{~h} / \mathrm{L}$ vs. $\left.70.0 \pm 12.0 \mathrm{mg}^{*} \mathrm{~h} / \mathrm{L}, p=0.039\right)$. Also $\mathrm{C}_{\max }$ levels were significantly lower in the obese individuals (mean $11.8 \pm 2.8 \mathrm{mg} / \mathrm{L} v s .18 .3 \pm 2.7 \mathrm{mg} * \mathrm{~h} / \mathrm{L}, p<0.001)$. No nephrotoxicity (based on the RIFLE criteria (26)) was observed in any participant.

\section{Pharmacokinetic Analysis}

A two-compartmental model with first-order elimination from the central compartment and a combined additional and proportional residual error model best described the data. IIV was implemented on clearance and central volume of distribution. Parameters of the structural model without covariates (base model) are shown in Table II.

Exploration using scatter plots of individual posthoc parameter estimates and IIV against different covariates indicated TBW, ABW and LBW as candidate covariates for central volume of distribution, and de-indexed MDRD, de-indexed CKD-EPI, CG-LBW, GFR, TBW and LBW for clearance. Figure 2 shows the individual posthoc parameter estimate for clearance versus the different candidate covariates, showing particularly clear relationships for GFR, MDRD and CKDEPI.

For central volume of distribution, TBW in a power function, LBW and ABW as linear covariates resulted in significant OFV drops $(-25.9,-23.9$ and -29.2 , respectively, all $p<0.001$ ). As TBW gave the best GOF (populations predicted versus observed concentrations) with the least bias especially in higher concentrations (i.e. $>12 \mathrm{mg} / \mathrm{L}$ ), TBW was selected over ABW $(p>0.05)$. Inclusion of TBW on central volume of distribution resulted in a reduction of IIV from $42.9 \%$ to $25.1 \%$ (Table II).

The results of the covariate implementation on CL are shown in Table III. Table III shows that implementation of de-indexed MDRD, de-indexed GKD-EPI, and GFR resulted in the largest reduction in $\mathrm{OFV}$, i.e. $-36.3,-32.8$ and -32.3 , respectively (all $p<0.001$ ). GOF plots for all covariates were comparable, although all models seemed to slightly underpredict tobramycin concentrations below $10 \mathrm{mg} / 1$ in the non-obese individuals (data not shown). The addition of TBW to de-indexed MDRD as covariate for clearance improved this underprediction, however the limited reduction in OFV (i.e. -3.4 in OFV, $p>0.05$ ) and only moderate improvement of GOF did not justify to include this extra parameter. Inclusion of de-indexed MDRD resulted in a reduction in IIV on clearance from $25.2 \%$ to $12.0 \%$ (Table II). Implementation of TBW instead of de-indexed MDRD, resulted in a power function on clearance with an estimated exponent of 0.42 , and was inferior to implementation of de-indexed MDRD (i.e. -10.3 versus -36.3 in OFV drop, $p<0.05$, and a resulting drop in IIV on CL of $25.2 \%$ to $20.6 \%$ versus $12.0 \%$, respectively). Implementation of the covariate relationship between TBW and clearance as found for gentamicin in similar study (17), i.e. a power relationship with an exponent of 0.729 , resulted in an even smaller drop in OFV (i.e. $-4.0, p<0.05$ ), with inferior GOF and only a very modest reduction in IIV from $25.2 \%$ to $23.4 \%$. As final model, de-indexed MDRD was selected as covariate on 
Table I Summary of Patient Characteristics

\begin{tabular}{|c|c|c|c|}
\hline & Morbidly obese $(n=20)$ & Non - obese $(n=8)$ & $P$ value \\
\hline Male/female & $9 / 11$ & $4 / 4$ & 0.57 \\
\hline Age (years) & $43.0[27-54]$ & $22.5[20-25]$ & $<0.001$ \\
\hline Total body weight (TBW, kg) & |37.8 [103-194] & $66.3[57-91]$ & $<0.001$ \\
\hline Lean body weight (LBW (19), kg) & $69.3[51-107]$ & $49.7[38-69]$ & 0.0029 \\
\hline Body Mass Index (BMI, kg/m²) & $41.9[36-53]$ & $22.2[19-25]$ & $<0.001$ \\
\hline Glomerular filtration rate based on 24-h urine collection (GFR, ml/min) & 163.3 [85-230] & $\mid 24.7[98-|4|]$ & 0.031 \\
\hline \multicolumn{4}{|l|}{ Estimated GFR } \\
\hline De-indexed Modification of Diet in Renal Disease (MDRD, ml/min) & $127.5[77-17 \mid]$ & |02.6 [9|-120] & 0.031 \\
\hline De-indexed Chronic Kidney Disease Epidemiology Collaboration (CKD-EPI, ml/min) & $138.0[78-17 \mid]$ & $109.4[10|-| 29]$ & 0.050 \\
\hline Cockcroft Gault with LBW (19) (obese) or TBW (non-obese) (CG-LBW, ml/min) & | $16.4[69-148]$ & $\mid 19.8[10 \mid-138]$ & 0.40 \\
\hline Tobramycin dose (mg) & $340[240-480]$ & $320[280-440]$ & 0.75 \\
\hline
\end{tabular}

Data shown as median [range] unless otherwise specified

clearance, since MDRD gave a significantly larger OFV reduction $(p<0.05)$ and better GOF compared to CKDEPI, and since in clinical practice a serum creatinine based eGFR such as MDRD is more readily available than 24-h urine based GFR.

The GOF plots of the final covariate model are shown in Fig. $\mathrm{S} 1$ in the supplemental material and show that the model described the data well. The parameters of the final model with confidence intervals based on the bootstrap analysis are shown in Table II together with final equations for clearance and central volume of distribution. The results from the boostrap analysis (Table II) indicate a good precision and stability of the final model. The prediction-variability corrected visual predictive check (pvcVPG) shown in Fig. 3 indicates good validity of the final model, with median and 5 th and 95th percentile of the observations being in concordance with the $95 \%$ confidence intervals of the simulations.

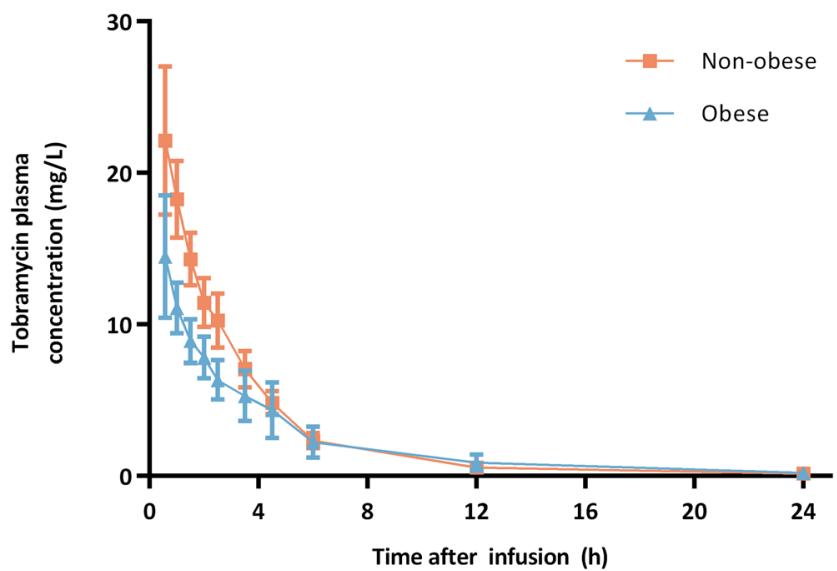

Fig. I Mean \pm SD tobramycin plasma concentrations versus time after end of infusion for obese (blue triangles, $n=20$, dose: $5 \mathrm{mg} / \mathrm{kg}$ lean body weight) and non-obese individuals (orange squares, $n=8$, dose: $5 \mathrm{mg} / \mathrm{kg}$ total body weight).

\section{Model-Based Simulations}

Figure 4 shows the individual (dots), median and interquartile range (boxplots) $\mathrm{AUC}_{24}$ values as obtained in the Monte Carlo simulations. Quantitative results are shown in Table S1 in the supplemental material. For individuals up to $100 \mathrm{~kg}$ (non-obese population), tobramycin was dosed as $5 \mathrm{mg} / \mathrm{kg}$ TBW. For obese individuals 100-190 kg, tobramycin was dosed using the nomogram depicted in Fig. 5, which is based on the relationship between clearance and MDRD as found in the final covariate model. The figure shows that when tobramycin is dosed as $5 \mathrm{mg} / \mathrm{kg}$ TBW, exposure increases with increasing body weight, with higher $\mathrm{AUC}_{24}$ values being observed in individuals with relatively low MDRD-values ( $<100 \mathrm{ml} / \mathrm{min}$, dark blue dots). Median $\mathrm{AUC}_{24}$ per weight subgroup of non-obese individuals when receiving $5 \mathrm{mg} / \mathrm{kg}$ TBW increases from around 50 to $80 \mathrm{mg}^{*} \mathrm{~h} / \mathrm{L}$ with increasing body weight. For individuals $>100 \mathrm{~kg}$, Fig. 4 shows that when a de-indexed MDRD-based dosing strategy is employed (using the nomogram in Fig. 5), no trend is visible with increasing body weight, with a median $\mathrm{AUC}_{24}$ tightly around $75 \mathrm{mg} * \mathrm{~h} / \mathrm{L}$. In case the $5 \mathrm{mg} / \mathrm{kg}$ TBW dosing strategy was employed in obese individuals, an increase in both the mean and variability (range) of exposures is observed, with a median of around $150 \mathrm{mg} * \mathrm{~h} / \mathrm{L}$ for obese individuals weighing around $190 \mathrm{~kg}$ (Fig. S2B in supplemental material). When the MDRD-based dosing strategy is used in non-obese individuals as well, no remaining trend in this population is found $(<100 \mathrm{~kg}$, Fig. S2A in supplemental material). Finally, when dosing was performed based on scaled body weight (i.e. using 0.42 as exponent for TBW (Table III)) or ABW, no clear trends are visible in median exposure across body weights similar to MDRD-based dosing (Fig. S2C and D in supplemental material). However, in contrast to MDRDbased dosing, these do yield a substantial reduction in 
Table II Population Pharmacokinetic Parameters of the Base and Final Tobramycin Model and Results of the Bootstrap Analysis

\begin{tabular}{|c|c|c|c|c|c|c|c|}
\hline & \multicolumn{2}{|c|}{ Base model (\%RSE) } & \multicolumn{2}{|c|}{ Final model (\%RSE) } & \multicolumn{3}{|c|}{ Bootstrap final model $(n=1000)$} \\
\hline & & & & & \multirow[b]{2}{*}{ Mean } & \multicolumn{2}{|c|}{ (95\% Confidence interval) } \\
\hline & & & & & & Lower & Upper \\
\hline$V_{c}(L)$ & 17.2 & $(7.3)$ & - & & & & \\
\hline \multicolumn{8}{|l|}{$\mathrm{V}_{\mathrm{c}}=\mathrm{V}_{\mathrm{c}_{70 \mathrm{~kg}}} *(\mathrm{TBW} / 70)$} \\
\hline$V_{c} 70 \mathrm{~kg}(\mathrm{~L})$ & - & & 10.6 & $(5.9)$ & 10.6 & 8.94 & 12.4 \\
\hline$C L(L / h)$ & 6.42 & $(4.3)$ & - & & & & \\
\hline \multicolumn{8}{|c|}{$C L=C_{M D R D} \mid 15 *(\mid+Z *(M D R D-|| 5)$} \\
\hline $\mathrm{CL}_{\text {MDRD }} 115(\mathrm{~L} / \mathrm{min})$ & - & & 6.33 & $(2.4)$ & 6.33 & 6.02 & 6.63 \\
\hline Z & - & & 0.00990 & $(3.9)$ & 0.0100 & 0.0880 & 0.0122 \\
\hline$V_{p}(L)$ & 4.24 & $(15)$ & 4.35 & $(5.6)$ & 4.41 & 2.84 & 5.98 \\
\hline $\mathrm{Q}(\mathrm{L} / \mathrm{min})$ & 6.4 & $(5.1)$ & 6.69 & $(1.6)$ & 6.77 & 2.63 & 10.91 \\
\hline \multicolumn{8}{|c|}{ Inter-individual variability (IIV, \%) } \\
\hline $\mathrm{V}_{\mathrm{c}}$ & 42.9 & $(9.3)$ & $24.9^{\mathrm{a}}$ & $(13)$ & 24.1 & 14.9 & 30.8 \\
\hline $\mathrm{CL}$ & 25.2 & $(14)$ & $12.0^{\mathrm{a}}$ & (13) & 11.7 & 7.90 & 14.5 \\
\hline \multicolumn{8}{|l|}{ Residual variability } \\
\hline Proportional error & 0.112 & $(12)$ & 0.116 & $(||)$ & 0.115 & 0.0880 & $0.14 \mid$ \\
\hline Additive error (mg/L) & 0.369 & $(13)$ & 0.346 & $(||)$ & 0.342 & 0.239 & 0.445 \\
\hline OFV & 351.7 & & 289.6 & & 276.6 & 185.9 & 367.2 \\
\hline
\end{tabular}

${ }^{a} \eta$-shrinkage in the final model is $8 \%$ for IIV on $C L$ and $6 \%$ for IN on $V_{c}$. CL Clearance from the central compartment, CLMDRD II5 Clearance from the central compartment for a person with a MDRD of I I $5 \mathrm{ml} / \mathrm{min}$, MDRD De-indexed Modification of Diet in Renal Disease (in $\mathrm{ml} / \mathrm{min}$ ), OFV Objective Function Value, $Q$ intercompartmental clearance between $V_{C}$ and $V_{P}$, RSE Relative standard error, TBW Total body weight in $\mathrm{kg}, V_{c}$ Central volume of distribution, $V_{c 70} \mathrm{~kg}$ Central volume of distribution for a $70 \mathrm{~kg}$ person

exposures within target in individuals with increased and decreased renal functions, respectively (Fig. S2C and D, Table S1).

\section{DISCUSSION}

In this report we studied the population pharmacokinetics tobramycin across body weights from 57 to $194 \mathrm{~kg}$ in individuals with a normal renal function. We show that with increasing body weight, tobramycin clearance is best predicted using a renal function estimate. In our data, this relationship between clearance and renal function was best described using de-indexed MDRD, although de-indexed CKD-EPI or GFR based on 24-h urine collection seem to lead to similar results. In order to reach the target exposure of $75 \mathrm{mg}^{*} \mathrm{~h} / \mathrm{L}$ in individuals of varying weights, model-based simulations (Fig. 4) were performed showing that in obese individuals $>100 \mathrm{~kg}$ tobramycin should be dosed using the proposed nomogram (shown in Fig. 5) based on the individuals de-indexed MDRD. Strong aspects of our study design are (1) the wide range of TBW in our study, including non-obese individuals and obese individuals up to $194 \mathrm{~kg}$, (2) the rich sampling procedure up to $24 \mathrm{~h}$ post-infusion and, (3) the use of a modelling and simulation strategy that is nowadays seen as the gold standard by regulatory authorities for approval of new dose regimens (28).

The influence of obesity on aminoglycoside clearance has been reported in some studies over the years (11-13,15,16,29). Although in general these studies found an increase in clearance with increasing body weight, their results have to be interpreted with caution since individuals in most of these studies were only moderate obese compared to present-day standards with average body weights around 85-105 kg with standard deviations of $\pm 12-18 \mathrm{~kg}(12,13,16)$. Moreover, analyses were often performed with sparse data collected up to only $8 \mathrm{~h}(11-13,15,16)$. These study designs limit the ability to properly assess drug clearance, particularly in view of the once every $24-\mathrm{h}$ dosing that is currently in practice. Only few studies report on covariates that can be used to adequately predict aminoglycoside clearance in obese individuals. One clinical study by Pai et al. in 497 subjects (with 91 obese patients), report that both gentamicin and tobramycin clearance could be best predicted using unadjusted eGFR formula (either MDRD or CKD-EPI) rather than de-indexed eGFR functions or the CG formula (15). Our study found better predictions for eGFR over the CG-formula as well, although we found that de-indexed eGFR is preferred over the 

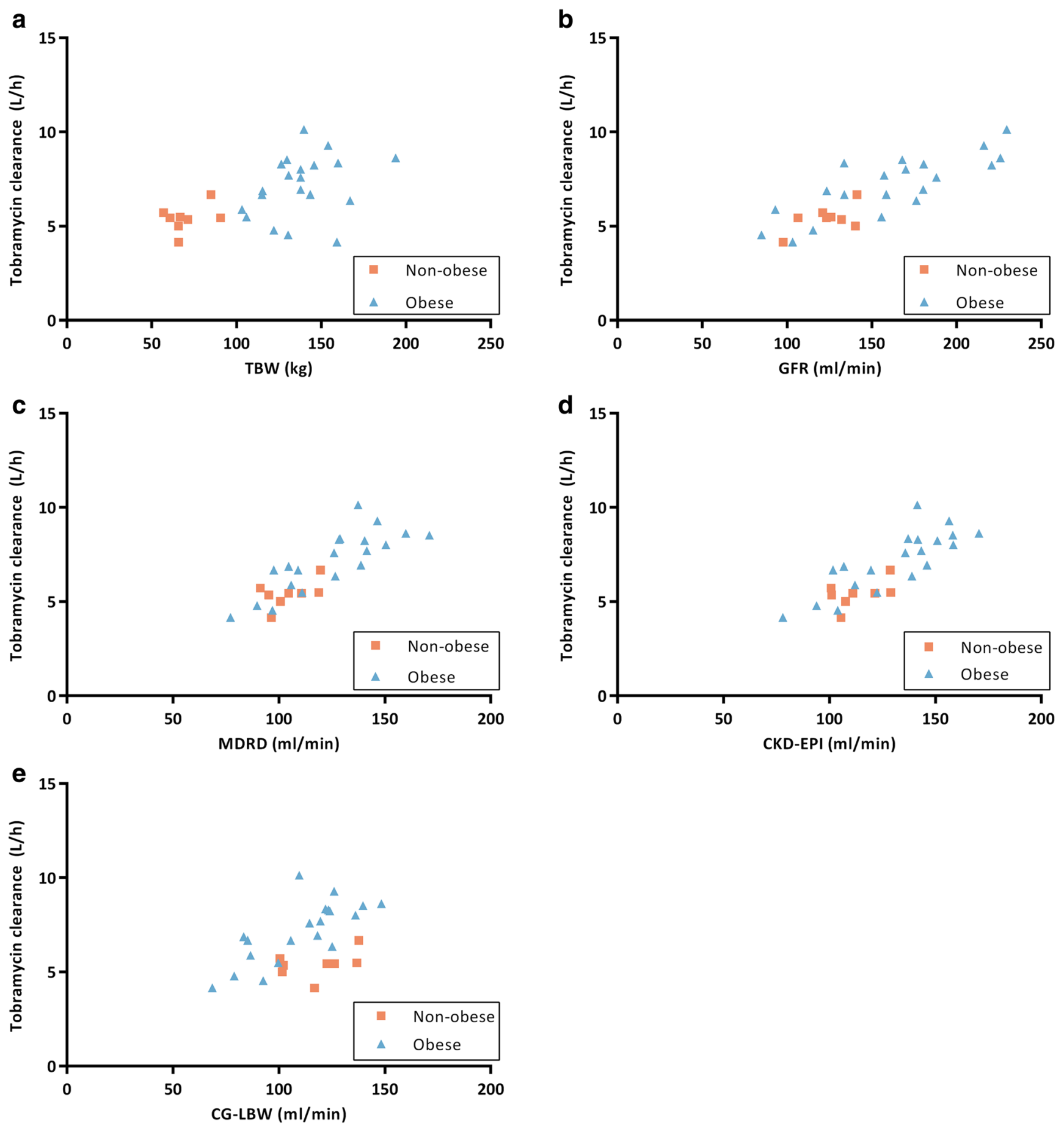

Fig. 2 Individual posthoc clearance values for tobramycin ( $n=28$, in L/h) versus (a) total body weight (TBW), (b) 24-h urine glomerular filtration rate (GFR), (c) eGFR based on de-indexed Modification of Diet in Renal Disease (MDRD), (d) de-indexed Chronic Kidney Disease Epidemiology Collaboration (CKD-EPI) and (e) Cockcroft-Gault using LBW in obese and TBW in non-obese (CG-LBW). Obese individuals are shown in blue triangles, non-obese individuals in orange squares.

unadjusted estimates. A possible explanation for this difference might be that Pai et al. had to rely on sparse data, potentially making it more difficult to estimate individual tobramycin clearances. In addition, the authors used Mosteller's equation for estimating BSA instead of the
Dubois and Dubois formula as employed in our analysis, which may result in some differences. However, our results did not change significantly when the Mosteller's equation was employed (data not shown). Lim et al. found in a retrospective study with 342 patients with $\sim 30 \%$ being obese, that 
Table III Impact of Different Covariates on Tobramycin Clearance (CL)

\begin{tabular}{|c|c|c|c|c|c|}
\hline Model & Parameter relationship (subpopulation) & X (exponential) / Z (linear) & Number of parameters & OFV & $\triangle \mathrm{OFV}^{\mathrm{a}}$ \\
\hline TBW on $V_{c}$ & - & - & 8 & 325.8 & (reference) \\
\hline TBW on CL & Exponential (all) & 0.42 & 9 & 315.6 & -10.3 \\
\hline TBW on $\mathrm{CL}^{\mathrm{b}}$ & Exponential (all) & 0.729 FIX & 9 & 321.8 & -3.96 \\
\hline MDRD on $\mathrm{CL}$ & Linear (all) & 0.0099 & 9 & 289.6 & -36.2 \\
\hline CKD-EPI on CL & Linear (all) & 0.0089 & 9 & 293.0 & -32.8 \\
\hline GFR on $C L$ & Linear (all) & 0.0055 & 9 & 293.5 & -32.3 \\
\hline CG-LBW on CL & Linear (obese) & 0.0069 & 9 & 315.9 & -9.88 \\
\hline
\end{tabular}

${ }^{a}$ OFV drop relative to reference model (base model with TBW on $V_{c}$ )

${ }^{\mathrm{b}}$ Covariate relationship for clearance and TBW as reported for gentamicin in similar study ( I7)

CG-LBW Cockcroft Gault using lean body weight for obese and total body weight for non-obese individuals, CKD-EPI De-indexed chronic Kidney Disease Epidemiology Collaboration, CL Clearance, GFR Glomerular Filtration Rate based on 24-h urine collection, OFV Objective Function Value, MDRD De-indexed modification of Diet in Renal Disease, TBW Total body weight, $V_{c}$ central volume of distribution

de-indexed eGFR outperformed their unadjusted counterparts in predicting aminoglycoside clearance (29). Leader et al. reported that $\mathrm{ABW}$ used in the $\mathrm{CG}$ equation is the best predictor for gentamicin clearance. Since this is an older study, no information is available on the performance of the eGFR formulas (12). Some other papers looked directly into predicting GFR in the obese population. These studies might be of relevance for our study, since in healthy adults, tobramycin clearance is shown to be primarily mediated through glomerular filtration (30). These papers generally agree that GFR can be best predicted using the de-indexed form of MDRD or CKD-EPI $(31,32)$, or the CG formula with LBW or ABW $(18,33,34)$. These conclusions are in line with our results, but should be translated to tobramycin clearance with caution
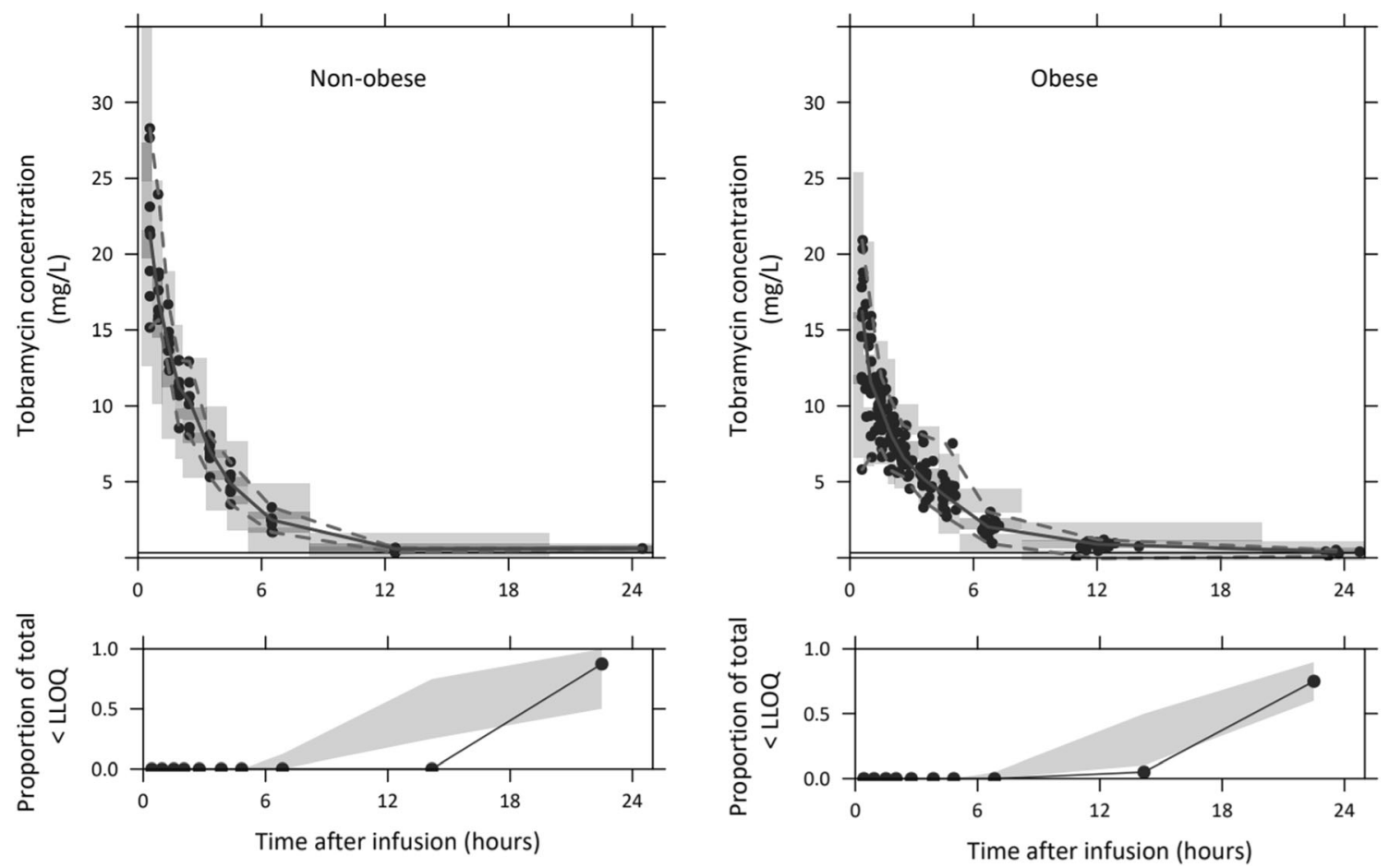

Fig. 3 Prediction- and variability-corrected visual predictive checks (pvcVPC) of the final model for non-obese (upper left panel) and obese (upper right panel) individuals ( $n=1000$ simulations). The observed concentrations are shown as black circles, with median, 2.5th and 97.5th percentiles of the observed data as solid line, lower dashed line and upper dashed line, respectively. The grey shaded areas depict the $95 \%$ confidence intervals of the median (dark grey) and 2.5th and 97.5th percentiles (light grey) of predicted concentrations. Lower panels show the observed proportion below LLOQ (black dots), where shaded areas depict the $95 \%$ confidence interval of these proportion based on the simulated concentrations. 


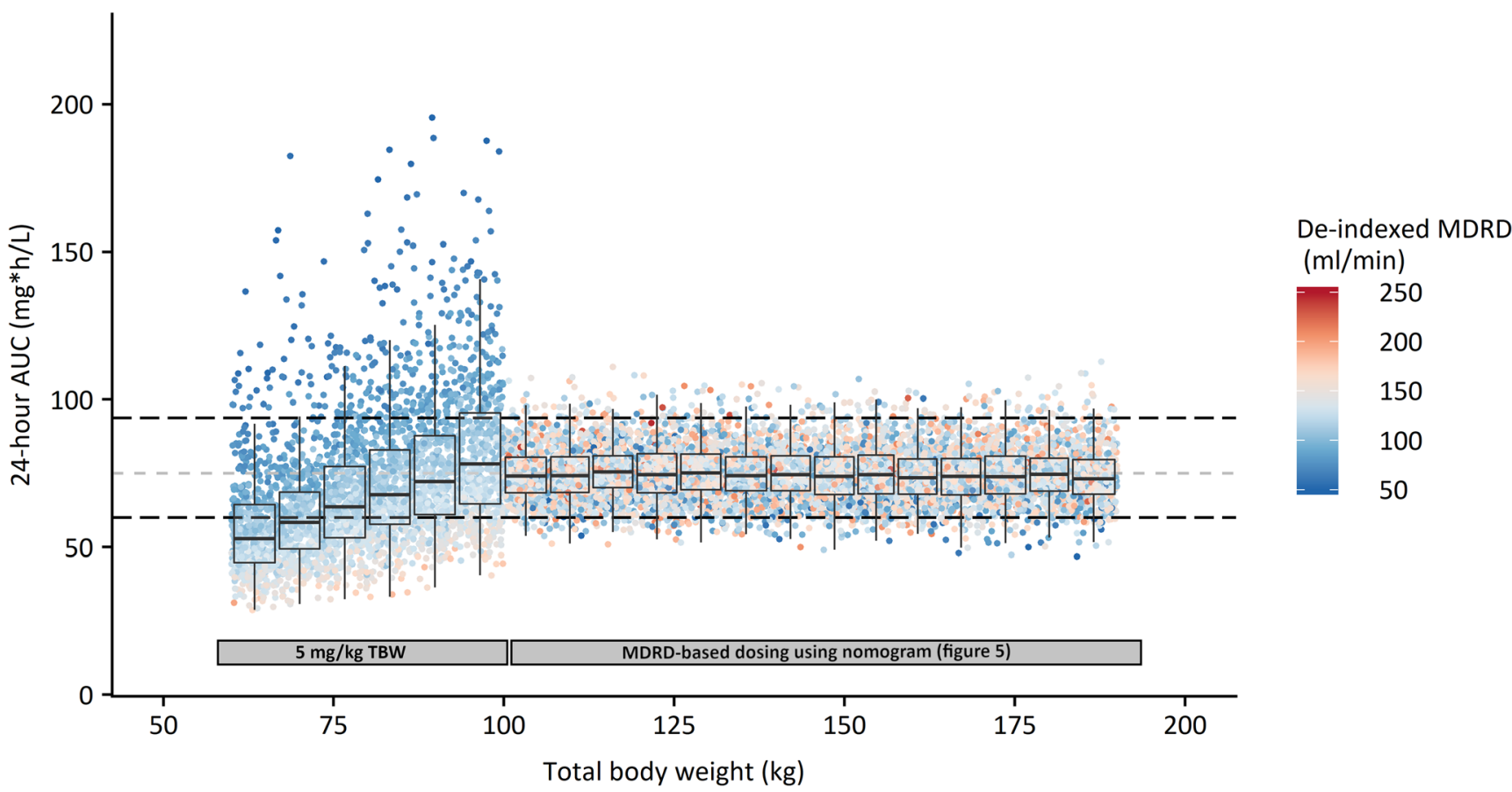

Fig. 4 Monte Carlo simulations ( $n=9.993$ ) for individuals $<100 \mathrm{~kg}$ receiving one IV dose of $5 \mathrm{mg} / \mathrm{kg}$ total body weight (TBW) tobramycin, and individuals $>100 \mathrm{~kg}$ received a MDRD-based tobramycin dose using the nomogram in Fig. 5. Each dot represents the $\mathrm{AUC}_{24}$ (in mg*h/L) of one individual in the dataset, where the color shows the de-indexed MDRD in $\mathrm{ml} / \mathrm{min}$ (calculated as MDRD * body surface area (BSA)/l.73) of this individual (ranging from dark blue to dark red with increasing MDRD). The boxplots represent median and interquartile range of $A \cup C_{24}$ values within a specific total body weight subgroup. The grey dashed line shows the target $\mathrm{AUC}_{24}$ of $75 \mathrm{mg}$ *h/ , black dashed lines show the 80-125\% range (EMA acceptance criteria for bio-equivalence studies (27)) relative to this target value. AUC Area under the curve, MDRD Modification of Diet in Renal Disease TBW Total body weight.

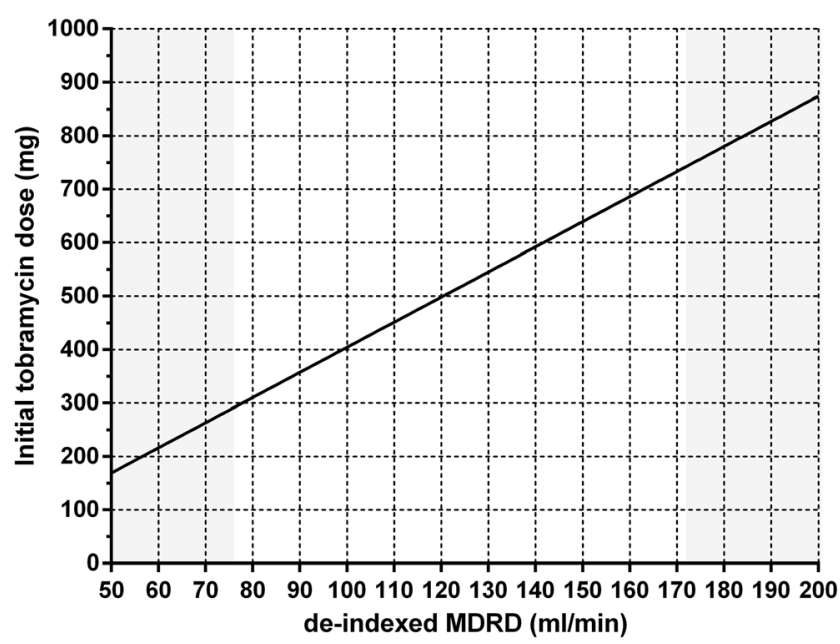

Fig. 5 Dosing nomogram for tobramycin dose (in $\mathrm{mg}$ ) based on the final tobramycin population PK model in non-obese and obese patients with body weights ranging from 57 to $194 \mathrm{~kg}$ and de-indexed MDRD values (calculated as MDRD * body surface area (BSA)/l.73) ranging from 77 to $171 \mathrm{ml} / \mathrm{min}$, aiming for an $\mathrm{AUC}_{24}$ of $75 \mathrm{mg}$ *h/ $\mathrm{L}$. The recommended tobramycin dose is calculated using equation: Dose $(\mathrm{mg})=\mathrm{AUC}_{24}$, target $\times 6.33 \times(1+$ $0.0099 \times(M D R D-115))$. where $A \cup C_{24, \text { target }}$ represents the target $\mathrm{AUC}_{24}$ in $\mathrm{mg}$ *ैh/L of 75 and MDRD represents the de-indexed MDRD in $\mathrm{ml} / \mathrm{min}$. Since the PK data consists of MDRD values from 77 to $171 \mathrm{ml} / \mathrm{min}$, dose recommendations extrapolation to values outside these should be interpreted with caution (grey area in the nomogram). MDRD Modification of Diet in Renal Disease. since other (active) processes might be involved besides glomerular filtration when using GFR to predict clearance of a drug. In summary, it appears that most literature point to a renal function estimate to be most predictive for tobramycin clearance in obese individuals, although results from previous studies are conflicting as to how these renal function estimates should be corrected in obese individuals. The current study, with rich data collected in a wide range of body weights and (unimpaired) renal functions, in our opinion now definitively shows that de-indexed MDRD or CKD-EPI outperform body weight, the CG formula (using either TBW or LBW) and unadjusted renal function estimates in predicting tobramycin clearance in obese individuals.

Our results on tobramycin differ from results that we have found for gentamicin in a recently performed prospective pharmacokinetic study that studied a similar patient population in a similar study design (17). This study showed that the increase in gentamicin clearance was best described by TBW with an estimated allometric exponent of 0.73 . In contrast to tobramycin, renal function estimates (eGFR or GFR based on 24-h urine collection) were inferior to TBW in predicting gentamicin clearance, despite the fact that in both studies individuals with a similar distribution in body weights and renal function (all $>60 \mathrm{ml} / \mathrm{min}$ ) were included. Interestingly, this finding has been reported before by other studies, describing 
stronger correlations between eGFR and drug clearance for tobramycin than gentamicin $(15,29)$. To explain this difference between tobramycin and gentamicin, it could be hypothesized that transporters play a role. For gentamicin an increase in renal organic cation transporter 2 (OCT2) activity and consequently enhanced renal uptake has been reported that may contribute to increased gentamicin clearance in the obese. In an obese overfed mouse model, OCT2 activity increased with obesity, leading to increased renal accumulation of gentamicin (35). In addition, it is well established from studies with metformin, which is a well-known OCT2 substrate, that for OCT2 substrates drug clearance is influenced by altered OCT2 function. A human study showed that OCT2 genotypes associated with impaired activity led to a reduced apparent metformin clearance (CL/F) (36). Moreover, an increase in metformin $\mathrm{CL} / \mathrm{F}$ was seen in obese adolescents compared to non-obese children, possibly due to an increase in renal OCT2-activity (37). In this light, the contrasting results on gentamicin and tobramycin clearance might be explained by a relatively higher dependence of gentamicin on OCT2mediated renal uptake in favor of glomerular filtration. Although to our best knowledge, this never has been properly studied, this hypothesis is further substantiated by the observation that tobramycin accumulates less in the kidney compared to gentamicin and therefore might be less nephrotoxic (38). Further (preclinical) research seems warranted to clarify these differences between tobramycin and gentamicin PK based on the current study results.

An important question is what the target $\mathrm{AUC}_{24}$ is when treating patients with tobramycin. An $\mathrm{AUC}_{24}$ of $75 \mathrm{mg} \mathrm{g}^{*} \mathrm{~h} \mathrm{~L}$ for pathogens with a MIC of $0,25-1 \mathrm{mg} / \mathrm{L}$ has been shown to be have the best balance between effectiveness and toxicity for aminoglycosides (5). Therefore, we provided a nomogram that can be used to determine the initial tobramycin dose for obese individuals based on the patient's de-indexed MDRD targeting an $\mathrm{AUC}_{24}$ of $75 \mathrm{mg}^{*} \mathrm{~h} / \mathrm{L}$ (Fig. 5). When this dose strategy is employed in the obese, a stable median $\mathrm{AUC}_{24}$ up to $190 \mathrm{~kg}$ without trends can be expected. In addition, outer ranges lie around $\sim 75 \%$ to $\sim 125 \%$ relative to the target of $75 \mathrm{mg}^{*} \mathrm{~h} / \mathrm{L}$ (absolute 95\% confidence interval of 57.4-93.5 and 56.9-92.8 $\mathrm{mg} * \mathrm{~h} / \mathrm{L}$ for non-obese and obese individuals, respectively, visualized in Fig. 4). This is acceptable, considering the acceptance range of $80-125 \%$ as specified by the European Medicines Agency (EMA) for bio-equivalence studies (27). In contrast, when a $5 \mathrm{mg} / \mathrm{kg}$ TBW dose regimen is employed in obese individuals, the $95 \%$ confidence intervals lie between $22.2 \mathrm{mg} * \mathrm{~h} / \mathrm{L}$ and $184.1 \mathrm{mg} * \mathrm{~h} / \mathrm{L}$, corresponding to $30 \%$ to $246 \%$ relative to the target $\mathrm{AUC}_{24}$. This high variability, which is most pronounced for the highest body weights of the obese population, can be explained by the fact that renal function is not taken into account in this strategy. Moreover, median $\mathrm{AUC}_{24}$ steadily increases with increasing body weight. In current daily practice, tobramycin is mostly dosed using ABW as is recommended by several papers, in order to maximize peak levels in obese individuals $(11,39,40)$. However, like with TBW-based dosing, this approach does not consider variation in renal function. As such, our simulations show that this approach leads to a substantial reduction in the proportion of patients having an AUC within the target $\mathrm{AUC}_{24}$ compared to using the dose nomogram for the obese population (43.9\% versus $93.6 \%$ ). Therefore, even though inadequate target concentrations can be picked up by therapeutic drug monitoring that is usually performed after a one or more dosages, we do not recommend to use TBW or ABWbased dose regimens in obese individuals.

A few remarks should be made regarding the proposed nomogram. First, the dose nomogram shows dose recommendations for de-indexed MDRD values ranging from 30 to $250 \mathrm{ml} / \mathrm{min}$. However, our PK-model is based on a dataset with MDRD values of 77 to $171 \mathrm{ml} / \mathrm{min}$. Dose recommendations outside of this MDRD-range should therefore be interpreted with caution in clinical practice. Second, the AUC-target of $75 \mathrm{mg}^{*} \mathrm{~h} / \mathrm{L}$ used in the nomogram is based on an AUC/MIC ratio of 75, with a corresponding MIC $\leq 1 \mathrm{mg} / \mathrm{L}$, as has been proposed earlier (5). However, it is known that the wild-type population of most gram-negatives extends to $2 \mathrm{mg} / \mathrm{L}$ (8). Therefore, higher dosages might be necessary to cover the whole range of pathogens with MIC values up to $2 \mathrm{mg} / \mathrm{L}$. Third, our study was specifically designed to obtain dose recommendations for obese individuals. A mg/kg-based dosing is already a widely accepted strategy for non-obese individuals. The proposed nomogram is expected to lead to an adequate exposure in the non-obese population as well (as shown in Fig. S3B in the supplemental material). Despite this, our simulations of a $5 \mathrm{mg} / \mathrm{kg}$ TBW dose (Fig. 4) show that in non-obese individuals, this strategy generally results in considerable variability. Last, after determining the initial tobramycin dose, we recommend that subsequent dosages should always be individualized by therapeutic drug monitoring, preferably with a limited sampling strategy in combination with model informed precision dosing based on Bayesian PK-software that is capable of translating the measured tobramycin concentrations to an individualized dose prediction (41).

Several limitations may apply to our study. First, we only included relatively healthy obese and non-obese individuals with an estimated renal function $>60 \mathrm{ml} / \mathrm{min} / 1.73 \mathrm{~m}^{2}$. Therefore, extrapolation of our study results to critically-ill patients with or without renal impairment should be done with caution, since critical illness can have an additional impact on PK. Secondly, obese study participants underwent bariatric surgery during the PK study, which might influence the PK results. However, since these surgeries in our hospital are very short $(<1 \mathrm{~h})$, and performed laparoscopically with minimal blood loss $(<50 \mathrm{~mL})$, we expect this impact to be negligible. 


\section{CONCLUSION}

In conclusion, we found that in non-obese and obese patients up to $194 \mathrm{~kg}$, tobramycin clearance shows an important relation with renal function estimates. In obese individuals, deindexed MDRD was superior over TBW in predicting tobramycin clearance. In order to yield similar exposure across body weights, we therefore propose that the tobramycin dose in individuals $>100 \mathrm{~kg}$ should be based on de-indexed MDRD. To aid the clinician in finding the optimal dose, we provide a dose nomogram that can be used to determine the correct initial tobramycin dose by integrating MDRD and target AUC.

\section{ACKNOWLEDGMENTS AND DISCLOSURES}

This study was funded by an institutional research grant number (ZonMW) 836,041,004. Dr. Brüggemann declares that he has no conflicts of interest with regard to this work. Outside of this work, he has served as consultant to and has received unrestricted research grants from Astellas Pharma Inc., F2G, Gilead Sciences, Merck Sharpe and Dohme Corp., and Pfizer Inc. All payments were invoiced by the Radboud University Medical Centre. All other authors declare no conflicts of interest. The authors would like to thank all study participants and Ingeborg Lange, Marieke van Donselaar, Angela Colbers, Brigitte Bliemer and Sylvia Samson for aiding in the inclusion of study participants.

Open Access This article is distributed under the terms of the Creative Commons Attribution 4.0 International License (http://creativecommons.org/licenses/by/4.0/), which permits unrestricted use, distribution, and reproduction in any medium, provided you give appropriate credit to the original author(s) and the source, provide a link to the Creative Commons license, and indicate if changes were made.

\section{REFERENCES}

1. GBD 2015 Obesity Collaborators, Afshin A, Forouzanfar MH, Reitsma MB, Sur P, Estep K, et al. Health effects of overweight and obesity in 195 countries over 25 years. N Engl J Med. 2017;377:13-27.

2. Smit C, De Hoogd S, Brüggemann RJM, Knibbe CAJ. Obesity and drug pharmacology: a review of the influence of obesity on pharmacokinetic and pharmacodynamic parameters. Expert Opin Drug Metab Toxicol. 2018;14:275-85.

3. Turnidge $\mathrm{J}$. Pharmacodynamics and dosing of aminoglycosides. Infect Dis Clin N Am. 2003;17:503-28.
4. Mouton JW, Jacobs N, Tiddens H, Horrevorts AM. Pharmacodynamics of tobramycin in patients with cystic fibrosis. Diagn Microbiol Infect Dis. 2005;52:123-7.

5. Drusano GL, Ambrose PG, Bhavnani SM, Bertino JS, Nafziger AN, Louie A. Back to the future: using aminoglycosides again and how to dose them optimally. Clin Infect Dis. 2007;45:753-60.

6. EUCAST. Gentamicin: Rationale for the EUCAST clinical breakpoint (version 1.2). 2009.

7. Pai MP, Rodvold KA. Aminoglycoside dosing in patients by kidney function and area under the curve: the Sawchuk-Zaske dosing method revisited in the era of obesity. Diagn Microbiol Infect Dis. 2014;78:178-87.

8. European Committee on Antimicrobial Susceptibility Testing (EUCAST). Data from the EUCAST MIC distribution website [nternet]. [cited 2019 Apr 4]. Available from: https://mic.eucast. org/. Accessed 4 April 2019

9. Prins JM, Büller HR, Kuijper EJ, Tange RA, Speelman P. Once versus thrice daily gentamicin in patients with serious infections. Lancet. 1993;341:335-9.

10. Rybak MJ, Abate BJ, Kang SL, Ruffing MJ, Lerner SA, Drusano GL. Prospective evaluation of the effect of an aminoglycoside dosing regimen on rates of observed nephrotoxicity and ototoxicity. Antimicrob Agents Chemother. 1999;43:1549-55.

11. Bauer LA, Edwards WAD, Dellinger EP, Simonowitz DA. Influence of weight on aminoglycoside pharmacokinetics in normal weight and morbidly obese patients. Eur J Clin Pharmacol. 1983;24:643-7.

12. Leader WG, Tsubaki T, Chandler MH. Creatinine-clearance estimates for predicting gentamicin pharmacokinetic values in obese patients. Am J Hosp Pharm. 1994;51:2125-30.

13. Schwartz SN, Pazin GJ, Lyon JA, Ho M, Pasculle AW. A controlled investigation of the pharmacokinetics of gentamicin and tobramycin in obese subjects. J Infect Dis. 1978;138:499-505.

14. Korsager S. Administration of gentamicin to obese patients. Int J Clin Pharmacol Ther Toxicol. 1980;18:549-53.

15. Pai MP, Nafziger AN, Bertino JS. Simplified estimation of aminoglycoside pharmacokinetics in underweight and obese adult patients. Antimicrob Agents Chemother. 2011;55:4006-11.

16. Sketris I, Lesar T, Zaske DE, Cipolle RJ. Effect of obesity on gentamicin pharmacokinetics. J Clin Pharmacol. 1981;21:288-93.

17. Smit C, Wasmann RE, Goulooze SC, Hazebroek EJ, van Dongen HPA, Burgers DMT, et al. A prospective clinical study characterizing the influence of morbid obesity on the pharmacokinetics of gentamicin: towards individualized dosing in obese patients. Clin Pharmacokinet 2019; Apr 24. https://doi.org/10.1007/s40262019-00762-4.

18. Demirovic JA, Pai AB, Pai MP. Estimation of creatinine clearance in morbidly obese patients. Am J Health Syst Pharm. 2009;66:642-8.

19. Janmahasatian S, Duffull SB, Ash S, Ward LC, Byrne NM, Green B. Quantification of lean bodyweight. Clin Pharmacokinet. 2005;44:1051-65.

20. Beal SL, Sheiner LB, Boeckmann A. NONMEM user's guide. San Francisco: University of California; 1999.

21. Keizer RJ, Karlsson MO, Hooker A. Modeling and simulation workbench for NONMEM: tutorial on Pirana, PsN, and Xpose. CPT Pharmacometrics Syst Pharmacol. 2013;2:e50.

22. R Core Team. R: A language and environment for statistical computing. 2015.

23. Beal SL. Ways to fit a PK model with some data below the quantification limit. J Pharmacokinet Pharmacodyn. 2001;28:481-504.

24. De Cock RFW, Allegaert K, Sherwin CMT, Nielsen EI, De Hoog M, Van Den Anker JN, et al. A neonatal amikacin covariate model can be used to predict ontogeny of other drugs eliminated through glomerular filtration in neonates. Pharm Res. 2014;31:754-67.

25. De Cock RFW, Allegaert K, Brussee JM, Sherwin CMT, Mulla H, De Hoog M, et al. Simultaneous pharmacokinetic modeling of 
gentamicin, tobramycin and vancomycin clearance from neonates to adults: towards a semi-physiological function for maturation in glomerular filtration. Pharm Res. 2014;31:2643-54.

26. Bellomo R, Ronco C, Kellum JA, Mehta RL, Palevsky P, Acute Dialysis Quality Initiative workgroup. Acute renal failure - definition, outcome measures, animal models, fluid therapy and information technology needs: the second international consensus conference of the acute Dialysis quality initiative (ADQI) group. Crit Care. 2004;8:R204-12.

27. Committee for Medicinal Products for human use (CHMP) European medicines agency (EMA). Guideline on the investigation of bioequivalence - CPMP/EWP/QWP/1401/98 Rev. 1. 2010.

28. European Medicines Agency (EMA). Guideline on the use of pharmacokinetics and pharmacodynamics in the development of antimicrobial medicinal products. 2016.

29. Lim AKH, Mathanasenarajah G, Larmour I. Assessment of aminoglycoside dosing and estimated glomerular filtration rate in determining gentamicin and tobramycin area under the curve and clearance. Intern Med J. 2015;45:319-29.

30. Naber KG, Westenfelder SR, Madsen PO. Pharmacokinetics of the aminoglycoside antibiotic tobramycin in humans. Antimicrob Agents Chemother. 1973;3:469-73.

31. Bouquegneau A, Vidal-Petiot E, Moranne O, Mariat C, Boffa J-J, Vrtovsnik F, et al. Creatinine-based equations for the adjustment of drug dosage in an obese population. BrJ Clin Pharmacol. 2016;81: 349-61.

32. Wuerzner G, Bochud M, Giusti V, Burnier M. Measurement of glomerular filtration rate in obese patients: pitfalls and potential consequences on drug therapy. Obes Facts. 2011;4:238-43.

33. Pai MP. Estimating the glomerular filtration rate in obese adult patients for drug dosing. Adv Chronic Kidney Dis. 2010;17:53-62.

34. Lim WH, Lim EM, McDonald S. Lean body mass-adjusted Cockcroft and gault formula improves the estimation of glomerular filtration rate in subjects with normal-range serum creatinine. Nephrology. 2006;11:250-6.

35. Gai Z, Visentin M, Hiller C, Krajnc E, Li T, Zhen J, et al. Organic cation transporter 2 overexpression may confer an increased risk of Gentamicin-induced nephrotoxicity. Antimicrob Agents Chemother. 2016;60:5573-80.

36. Yoon H, Cho H-Y, Yoo H-D, Kim S-M, Lee Y-B. Influences of organic cation transporter polymorphisms on the population pharmacokinetics of metformin in healthy subjects. AAPS J. 2013;15: 571-80.

37. van Rongen A, van der Aa MP, Matic M, van Schaik RHN, Deneer VHM, van der Vorst MM, et al. Increased metformin clearance in overweight and obese adolescents: a pharmacokinetic substudy of a randomized controlled trial. Paediatr Drugs. 2018;20: 365-74.

38. Schentag.JJ, Plaut ME, Cerra FB. Comparative nephrotoxicity of gentamicin and tobramycin: pharmacokinetic and clinical studies in 201 patients. Antimicrob Agents Chemother. 1981;19:859-66.

39. Wurtz R, Itokazu G, Rodvold K. Antimicrobial dosing in obese patients. Clin Infect Dis. 1997;25:112-8.

40. Pai MP, Bearden DT. Antimicrobial dosing considerations in obese adult patients. Pharmacotherapy. 2007;27:1081-91.

41. Nezic L, Derungs A, Bruggisser M, Tschudin-Sutter S, Krähenbühl S, Haschke M. Therapeutic drug monitoring of once daily aminoglycoside dosing: comparison of two methods and investigation of the optimal blood sampling strategy. Eur J Clin Pharmacol. 2014;70:829-37.

Publisher's note Springer Nature remains neutral with regard to jurisdictional claims in published maps and institutional affliations. 